\title{
Endothelial Dysfunction Induced by Chronic Psychological Stress: A Risk Factor for Atherosclerosis
}

\section{Hariane Côco $^{1,2 *}$ and Ana Maria de Oliveira ${ }^{2}$}

${ }^{1}$ Departamento de Farmacologia, Faculdade de Medicina de Ribeirão Preto, Universidade de São Paulo (USP), Avenida do Café s/n, Brazil ${ }^{2}$ Laboratório de Injúria Vascular, Faculdade de Ciências Farmacêuticas de Ribeirão Preto, USP; Avenida do Café s/n, Monte Alegre, Brazil

\begin{abstract}
The exposure to psychological stress can increase the risk of cardiovascular disease due to impaired endothelial function. Mediators such as glucocorticoids, catecholamines, angiotensin II and/or pro-inflammatory cytokines, induced by stress, can contribute to endothelial dysfunction due to increased levels of oxidative stress. The endothelial dysfunction induces reduced expression and/or endothelial nitric oxide synthase enzyme functionality, as well as the impairment of the actions triggered by its metabolite, nitric oxide. Chronic psychological stress leads to atherosclerosis development, which has an endothelial dysfunction at early stages. The oxidative damage and inflammatory mediators, induced by chronic psychological stress, play a key role in this process. Furthermore, chronic psychological stress can contribute to the formation of unstable atherosclerotic lesions as a result of immune system cells accumulation and molecules adhesion, leading to thrombosis and cardiac complications. According to what was stated above, we aim to discuss about the endothelial function impairment mediated by psychological stress, and the involvement of mediators such as glucocorticoids, catecholamines, angiotensin II and pro-inflammatory cytokines in this response. This review covers current advancements to understand how chronic psychological stress could lead to atherosclerosis development.
\end{abstract}

Keywords: Chronic psychological stress; Oxidative stress; Inflammatory factors; Endothelial dysfunction; Atherosclerosis

\section{Introduction}

Chronic exposure to stressful situations is associated with increased risk of cardiovascular diseases [1], including acute myocardial infarction [2], coronary artery disease [3-5], myocardial ischemia [6] and atherosclerosis $[7,8]$. The odds for acute myocardial infarction have increased more than twice in individuals who were exposed to psychosocial factors associated with exposure to multiple risk factors, such as hypertension, smoking, diabetes mellitus and obesity, in comparison to those who were not exposed to psychosocial factors [2]. The increased risk to acute myocardial infarction triggered by exposure to psychosocial factors was independent of gender, age or geographic distribution [2]. The greatest severity of stress-triggered depressive symptoms was related to reduce long-term survival after myocardial infarction [9]. It has been studied the relation between panic disorder, considered as part of the multifactorial clinical picture of mental stress, and coronary artery disease [10]. Soares-Filho et al. [11] discussed about the endothelial dysfunction and microvascular disease present in mental stress response and suggest that mechanisms from the perspective of endothelial dysfunction and microvascular disease might be involved in mental stress. All these findings suggest that the pathophysiologic effects of psychological stress may trigger the development of cardiac events.

\section{Psychological Stress and Endothelial Dysfunction}

Stress is a state of disharmony or threat to homeostasis, caused by psychological, environmental or physiological stressors, which lead to specific or generalized adaptive responses [12]. The biological systems respond to stressful stimuli and attempt to develop an adaptation process to these alterations induced by stressors [13]. An imbalance between stressors and response against it leads to maladaptation, which can be a promoter of various diseases, including cardiovascular diseases [13].
The responses to stressors are initiated by the sensory system stimulation, leading to the activation of several adaptive mechanisms, such as hypothalamic-pituitary-adrenal (HPA) axis and the sympathetic nervous system [14]. Mental stress stimulates the paraventricular nucleus of the hypothalamus, where corticotropin-releasing factor is produced and released, which acts on pituitary in order to release the adrenocorticotropin hormone, resulting in the releasing of glucocorticoids from the adrenal glands cortex [15]. Increased serum levels of glucocorticoids can lead to biological alterations in the body, including the development of some psychological disorders and systemic diseases, such as inflammatory, metabolic and autoimmune diseases [16-19]. Abnormalities in HPA axis functionality have been associated with cardiovascular risk factors [20,21]. Additionally, the sympathetic nervous system activation triggers increased vascular tone, myocardial oxygen consumption, platelets activation, as well as activation of renin-angiotensin system, which results in endothelial dysfunction, and hence, the development of cardiovascular diseases [13].

Endothelial dysfunction is mediated by impaired nitric oxide (NO). The endothelium-derived NO plays a main role in maintaining vascular homeostasis, and it is an important mediator of vasodilation. The biosynthesis of NO results from the oxidation from L-arginine to L-citrulline, catalyzed by the nitric oxide synthase enzyme (NOS),

*Corresponding author: Hariane Côco, Departamento de Farmacologia, Faculdade de Medicina de Ribeirão Preto, Universidade de São Paulo (USP) Avenida do Café s/n, Monte Alegre, 14040-903, Ribeirão Preto, SP, Brazil, Tel: +55 163315 4706; E-mail: harianec@yahoo.com.br

Received November 18, 2015; Accepted December 11, 2015; Published December 17, 2015

Citation: Côco H, Oliveira AM (2015) Endothelial Dysfunction Induced by Chronic Psychological Stress: A Risk Factor for Atherosclerosis. Cardiovasc Pharm Open Access 4: 168. doi:10.4172/2329-6607.1000168

Copyright: @ 2015 Côco H, et al. This is an open-access article distributed under the terms of the Creative Commons Attribution License, which permits unrestricted use, distribution, and reproduction in any medium, provided the original author and source are credited. 
in presence of tetrahydrobiopterin cofactor $\left(\mathrm{BH}_{4}\right)$. There are three isoforms of NOS, one induced by immunological stimuli (iNOS) and two of them constitutive, endothelial NOS (eNOS) and neuronal NOS (nNOS) [22]. After it is produced, NO diffuses from the endothelium into the vascular smooth muscle and lead to vascular relaxation. This fact occurs because NO binds to the soluble guanylyl cyclase heme (sGC) enzyme and produces cyclic guanosine monophosphate (cGMP), which activates protein kinase (PK) G, triggering a cellular signaling cascade, which decreases the concentration of cytosolic calcium, and hence, the vascular relaxation [23].

Considering the importance of $\mathrm{NO}$ on vascular homeostasis regulation, an imbalance between reduced NO bioavailability and/ or increased endothelium-derived contractile factor could lead to endothelial dysfunction [24]. The lower NO bioavailability can result from the consumption of NO by reactive oxygen species (ROS), reduced eNOS expression [25] and lack of eNOS substrate or cofactors [26]. The latter plays an important role in the enzymatic decoupling, resulting in ROS production instead of NO.

Oxidative stress is defined as an imbalance between antioxidant defenses, such as superoxide dismutase (SOD) and catalase, and ROS, such as superoxide anion $\left(\mathrm{O}_{2}^{-}\right)$and hydrogen peroxide $\left(\mathrm{H}_{2} \mathrm{O}_{2}\right)$ [27]. Many enzymatic systems can produce $\mathrm{O}_{2}^{-}$, as decoupled NOS [28], cyclooxygenase (COX) [29] and nicotinamide-adenine-dinucleotidephosphate enzymatic complex (NADPH oxidase). The latter is the biggest source of ROS in vascular cells $[30,31]$.

Recent studies suggest that psychological stress triggers endothelial dysfunction [32]. Patients with mild symptoms of depression or stress, showed high depression or stress score associated to reduced brachial artery flow-mediated dilation, which suggests the relationship between stress and endothelial dysfunction [33]. Mice exposed to chronic unpredictable stress, for 8 weeks, showed impaired endotheliumdependent relaxation in aorta [34]. The relaxation response, induced by acetylcholine (ACh), was also significantly reduced in thoracic aortas from rats exposed to chronic unpredictable stress for 3 weeks [35]. These results suggest reduced production and/or bioavailability of NO after stress. Additionally, chronic unpredictable stress increased the potency of phenylephrine (PE) in thoracic aortic rings from rats, suggesting that the possible lack of NO bioavailability could contribute to hyper reactivity of agonists and impair the vascular function $[35,36]$.

According to the previously described findings, the acute restraint stress reduced vascular and plasma levels of NO metabolites, nitrate and nitrite, and increased $\mathrm{O}_{2}{ }_{2}^{-}$levels in rats' aorta, suggesting endothelial dysfunction as a result of oxidative stress, induced by stressful situations [37]. Acute restraint stress also increased the reactivity to $\mathrm{PE}$ in rats' aorta, and pre-incubation with L-NAME non-selective NOS inhibitor did not affect this response, suggesting that increased contraction induced $\mathrm{PE}$ in aorta from stressed rats may be due to impaired modulation of $\mathrm{NO}$ [37].

The mechanism in which stress induces endothelial dysfunction may involve important mediators, including glucocorticoids, catecholamines, angiotensin (Ang) II and pro-inflammatory cytokines. Treatment with antagonists of glucocorticoid receptors, mifepristone, improved the endothelium-dependent relaxation and the eNOS gene expression in mice's aorta, both impaired by chronic stress [38]. Mifrepristone also reduced ROS vascular levels, which were higher after stress. These findings suggest that glucocorticoid generation, induced by exposure to stress, impair endothelial function [38]. The exposure to dual challenge (physical and psychological stress) increased the levels of noradrenaline and oxidative stress, as well as the noradrenaline area- under-the-curve was positively correlated with peak oxidative stress marker 8-isoprostane, suggesting that the increased noradrenaline levels may be involved with increased oxidative stress after physical and psychological stress. This fact could contribute to endothelial dysfunction [39].

The increased sympathetic activity, induced by stress, contributes to the activation of the renin-angiotensin system $[40,13]$. Stress-induced sympathetic activation of beta-adrenergic receptors in the kidneys increases renin formation and subsequently, the generation and release of Ang II into circulation [41]. Plasma renin activity and/or Ang II levels are increased in subjects exposed to situations of fear, novelty [42] and to hot temperatures [43]. In rats, the activation of renin-angiotensin system also increased in response to chronic restraint stress $[44,45]$. Chies et al. [46] demonstrated that rats exposed to swimming stress presented reduction in the contractile response induced by Ang II in thoracic aortas and mesenteric arteries, suggesting that the exposure to stress alters the vascular reactivity to Ang II.

The increase in Ang II levels, induced by psychological stress, influences endothelial function. The chronic restraint stress reduced endothelium-dependent relaxation, plasma levels of stable NO metabolite (nitrate and nitrite) and vascular eNOS gene expression, as well as increased oxidative stress [45]. The treatment with $\mathrm{AT}_{1}$ receptors antagonist, losartan, or angiotensin-converting enzyme inhibitor, ramipril, blunted these responses, suggesting that the involvement of $\mathrm{AT}_{1}$ receptors and Ang II in the endothelial dysfunction, induced by chronic restraint stress [45].

The inflammatory process can also trigger endothelial dysfunction. Treatment with etanercept, an anti-TNF- $\alpha$, improved the endotheliumdependent relaxation and the eNOS protein expression, both impaired in aorta from rats, after exposure to chronic unpredictable stress [47]. These results show that TNF- $\alpha$ plays an important pathophysiological role in the endothelial dysfunction induced by stress.

Thus, abnormalities in functionality of HPA axis or increased sympathetic activity, and hence, increased levels of glucocorticoids and catecholamines, lead to higher levels of oxidative stress and inflammation, which are considered risk factors for endothelial dysfunction. Consequently, these factors may increase the risk of cardiovascular diseases development, including atherosclerosis [48].

\section{Psychological Stress as a Risk Factor for Atherosclerosis}

Atherosclerosis is a chronic disease characterized by formation of atheroma in the medium and large artery wall. The development and progression of atherosclerotic lesions are triggered by endothelial dysfunction, followed by recruitment of leukocytes to the vascular wall, which modifies the structure of low density lipoproteins (LDL) deposited on the endothelial layer. Among the LDL modification, the oxidation induced by ROS results in formation of oxidized LDL and hence, these modified LDL suffer phagocytosis by macrophages and accumulate in vascular cells. These alterations lead to formation of foam cells, which can initiate atherosclerotic lesions [49].

The development of atherosclerosis associated with psychological stress has been studied since 1983. Chronic psychosocial stress increased area occupied by intimal lesion in monkeys coronary artery, in the absence of other risk factors for atherosclerosis, such as alterations in lipid levels, blood pressure or glucose. These findings suggest that psychosocial stress causes atherosclerotic lesions in nonhuman primates without any traditional risk factor [7].

The impaired endothelial function induced by stress triggers 
pro-atherogenic effects. Concentration-effect curves showed greater PE potency in endothelium-intact aorta from rats, after exposure to chronic unpredictable stress [36]. The pre-incubation with NOS inhibitor, L-NAME, did not alter this response, suggesting that, the increased PE potency induced by stress can be related to decreased production and/or bioavailability of endothelial NO [36]. These alterations were associated with an increase in the total intima-mediathickness of the carotid artery [36], a parameter that indicates a risk factor for cardiovascular disease [50].

Chronic restraint stress has increased the levels of oxidative stress in aortas from apolipoprotein (Apo) E-deficient mice, resulting in increased expression of LOX-1, a receptor to the modified LDL [51]. Treatment with EUK-8, a mimetic of SOD and catalase enzymes, reversed the increased LOX-1 expression, suggesting that chronic restraint stress upregulates vascular LOX-1 expression, through a mechanism that involves increased oxygen free radicals generation, which may contribute to the development of pro-atherosclerotic conditions [51]. In addition, mice exposure to chronic restraint stress increased oxidative stress levels in the liver, kidney and heart, and this response was associated with reduced activity of liver antioxidant enzymes [52]. These changes were accompanied by the formation of foam cells and accumulation of lipids in the mice's aorta, suggesting that chronic stress leads to oxidative stress and also to initiate atherosclerotic lesions [52].

Atherosclerotic lesions are characterized by sub-endothelial retention of inflammatory cells, whose adhesion is mediated by adhesion molecules, such as vascular (VCAM-1) and intercellular cell adhesion molecules (ICAM-1). On the other hand, the leukocyte migration is induced by chemokine expression in the vascular wall, such as monocyte chemotactic protein (MCP-1). The pro-inflammatory cytokines, including TNF- $\alpha$, interleukin (IL) -1 and IL-6, secreted by activate inflammatory cells, maintain an inflammatory vascular environment and thus, perpetuate atherosclerosis [53].

Chronic stress plays a critical role in the induction of vascular inflammation and progression of atherosclerotic lesions [54-57]. The chronic unpredictable stress increased plasma TNF- $\alpha$ levels and its expression in rabbits' aorta, as well as infiltration of macrophage and lipid accumulation in the sub-endothelial space. Such characteristics are similar to initial stages of atherosclerosis [57]. Vascular smooth muscle cells treated with serum from stressed rabbits showed increase in the expression of MCP-1, ICAM-1, phosphorylation of mitogenactivated protein kinase (MAPKs), MAPKp38 and c-jun N-terminal kinase (JNK) [57]. The MAPKs signaling pathway regulates the processes of cell differentiation and proliferation, protein expression to leukocyte adhesion and it is involved in the atherosclerotic process. Pro-inflammatory effects induced by chronic stress were partially blocked by anti-TNF- $\alpha$ antibody, MAPKp38 and JNK inhibitors, suggesting that TNF- $\alpha$, MAPKp38 and JNK activation can play a critical role in the pro-inflammatory and pro-atherogenic effects after chronic stress [57].

The rupture of atherosclerotic plaque is considered one of the main causes of cardiovascular complications [53]. A plate with large lipids pool, a thin fibrous cap and many inflammatory cells is prone to suffer rupture when an external stimulus occurs, such as psychological stress. Chronic unpredictable stress led to a plaque rupture and increased plaque size in brachiocephalic arteries from Apo E-deficient mice, as well as accumulation of inflammatory cells and adhesion molecule in the atherosclerotic lesions. These data suggest that the alterations, mediated by chronic stress on plate composition, can promote instability and contribute to its rupture [54].

The participation of adrenergic receptors in the inflammation, induced by stress-associated atherosclerosis, has been studied. Apo E-deficient mice exposed to social disruption stress showed a correlation between stress-score and median aortic plaque area, as well as increased levels of IL-6 pro-inflammatory cytokine. These observations suggest that psychological stress is involved with the atherosclerosis progression, and the release of IL- 6 may be a potential mechanism for the atherosclerosis development [56]. The selective beta1-adrenoceptor blockade with metoprolol prevented the increased IL-6 levels in plasma, induced by social disruption stress, suggesting that the sympathetic nervous system may mediate the release of IL-6 and lead to a progression of atherosclerotic lesions [56]. The inhibition of alfa/beta-adrenergic receptors reduced the lipid deposits, induced by chronic restraint stress, in aortas from Apo E-deficient mice, suggesting that the stress-induced atherosclerotic lesions are associated with activation of adrenergic receptors [58].

Increased activity of the sympathetic nervous system reduced the CXCL12 chemokine expression in the bone marrow from mice, after exposure to chronic unpredictable stress [59]. One of the main functions of this chemokine is to inhibit the migration and proliferation of hematopoietic stem cell, and hold the neutrophils inside the bone marrow. Thus, the reduced expression of CXCL12 led to proliferation of hematopoietic stem cells, and increased production of neutrophils and monocytes. These events resulted in increased release of inflammatory leukocytes into circulation, and hence, the development of inflammatory plaque [59]. The treatment of stressed mice with beta3-selective receptor antagonist, SR 59230A, has limited the progression of atherosclerotic lesions, which supports the idea that the sympathetic nervous system, signaling via beta 3 -adrenergic receptors activation, has targeted the CXCL12 chemokine in the bone marrow, and it could be explored as potential therapeutic approach in the stress-induced atherosclerosis [59].

\section{Final Considerations}

Psychological stress activates the sympathetic nervous system, renin-angiotensin system and the HPA axis, triggering increased levels of catecholamines, glucocorticoids and Ang II, respectively. All these mediators can promote endothelial dysfunction due to the increase in oxidative stress and circulating inflammatory mediators, as well as reduced NO production or bioavailability. These alterations lead to vascular inflammation and, consequently, the development of atherosclerosis. Furthermore, TNF-alpha and MAP kinases signaling pathway played a potential role in the inflammatory process, as well as the adrenergic receptors and CXCL12 chemokine that are involved with hematopoietic activation and the development of atherosclerotic lesions.

\section{References}

1. Steptoe A, Kivimäki M (2012) Stress and cardiovascular disease. Nat Rev Cardiol 9: 360-370.

2. Yusuf S, Hawken S, Ounpuu S, Dans T, Avezum A, et al. (2004) Effect of potentially modifiable risk factors associated with myocardial infarction in 52 countries (the INTERHEART study): case-control study. Lancet 364: 937-952.

3. Rozanski A, Blumenthal JA, Kaplan J (1999) Impact of psychological factors on the pathogenesis of cardiovascular disease and implications for therapy. Circulation 99: 2192-2217.

4. Rozanski A, Blumenthal JA, Davidson KW, Saab PG, Kubzansky L (2005) The epidemiology, pathophysiology, and management of psychosocial risk factors in cardiac practice: the emerging field of behavioral cardiology. J Am Coll Cardiol 45: 637-651. 
5. Strike PC, Steptoe A (2004) Psychosocial factors in the development of coronary artery disease. Prog Cardiovasc Dis 46: 337-347.

6. Blumenthal JA, Jiang W, Waugh RA, Frid DJ, Morris JJ, et al. (1995) Mental stress-induced ischemia in the laboratory and ambulatory ischemia during daily life. Association and hemodynamic features. Circulation 92: 2102-2108.

7. Kaplan JR, Manuck SB, Clarkson TB, Lusso FM, Taub DM, et al. (1983) Social stress and atherosclerosis in normocholesterolemic monkeys. Science 220: 733-735.

8. Williams JK, Kaplan JR, Manuck SB (1993) Effects of psychosocial stress on endothelium-mediated dilation of atherosclerotic arteries in cynomolgus monkeys. J Clin Invest 92: 1819-1823.

9. Lespérance F, Frasure-Smith N, Talajic M, Bourassa MG (2002) Five-year risk of cardiac mortality in relation to initial severity and one-year changes in depression symptoms after myocardial infarction. Circulation 105: 1049-1053.

10. Soares-Filho GL, Arias-Carrión O, Santulli G, Silva AC, Machado S, et al. (2014) Chest pain, panic disorder and coronary artery disease: a systematic review. CNS Neurol Disord Drug Targets 13: 992-1001.

11. Soares-Filho GL, Machado S, Arias-Carrión O, Santulli G, Mesquita CT, et al (2014) Myocardial perfusion imaging study of $\mathrm{CO}(2)$-induced panic attack. Am J Cardiol 113: 384-388

12. McEwen BS (2000) The neurobiology of stress: from serendipity to clinical relevance. Brain Res 886: 172-189.

13. Inoue N (2014) Stress and atherosclerotic cardiovascular disease. J Atheroscler Thromb 21: 391-401.

14. Black PH (2002) Stress and the inflammatory response: a review of neurogenic inflammation. Brain Behav Immun 16: 622-653.

15. Ulrich-Lai YM, Herman JP (2009) Neural regulation of endocrine and autonomic stress responses. Nat Rev Neurosci 10: 397-409.

16. McEwen BS, Biron CA, Brunson KW, Bulloch K, Hambers WH, et al. (1997) The role of adrenocorticoids as modulators of immune function in health and disease: neural, endocrine and immune interactions. Brain Res Brain Res Rev 23: $79-133$.

17. Andersson IM, Lorentzen JC, Ericsson-Dahlstrand A (2000) Analysis of Adrenocortical Secretory Responses during Acute and Prolonged Immune Stimulation in Inflammation-Susceptible and -Resistant Rat Strains. J Neuroendocrinol 12: 1096-1104.

18. Bosch JA, Berntson GG, Cacioppo JT, Dhabhar FS, Marucha PT (2003) Acute stress evokes selective mobilization of $T$ cells that differ in chemokine receptor expression: a potential pathway linking immunologic reactivity to cardiovascular disease. Brain Behav Immun 17: 251-259.

19. Windle RJ, Wood SA, Kershaw YM, Ligthman SL, Ingram CD, et al. (2001) Increased Corticosterone Pulse Frequency During Adjuvant-Induced Arthritis and its Relationship to Alterations in Stress Responsiveness. J Neuroendocrino 13: $905-911$.

20. Girod JP, Brotman DJ (2004) Does altered glucocorticoid homeostasis increase cardiovascular risk? Cardiovasc Res 64: 217- 226

21. Hamer M, O'Donnell K, Lahiri A, Steptoe A (2010) Salivary cortisol responses to mental stress are associated with coronary artery calcification in healthy men and women. Eur Heart J 31: 424-429.

22. Moncada S, Palmer RM, Higgs EA (1991) Nitric oxide: physiology pathophysiology, and pharmacology. Pharmacol Rev 43: 109-142.

23. Rapoport RM, Murad F (1983) Agonist-induced endothelium-dependent relaxation in rat thoracic aorta may be mediated through cGMP. Circ Res 52 352-357

24. Lerman A, Burnett JC Jr (1992) Intact and altered endothelium in regulation of vasomotion. Circulation 86: III12-19.

25. Wilcox JN, Subramanian RR, Sundell CL, Tracey WR, Pollock JS, et al. (1997) Expression of multiple isoforms of nitric oxide synthase in normal and atherosclerotic vessels. Arterioscler Thromb Vasc Biol 17: 2479-2488.

26. Pou S, Pou WS, Bredt DS, Snyder SH, Rosen GM (1992) Generation of superoxide by purified brain nitric oxide synthase. J Biol Chem 267: 2417324176

27. Schiffrin EL (2008) Oxidative stress, nitric oxide synthase, and superoxide dismutase: a matter of imbalance underlies endothelial dysfunction in the human coronary circulation. Hypertension 51: 31-32.

28. Satoh M, Fujimoto S, Haruna Y, Arakawa S, Horike H, et al. (2005) NAD(P)H oxidase and uncoupled nitric oxide synthase are major sources of glomerular superoxide in rats with experimental diabetic nephropathy. Am J Physiol Renal Physiol 288: F1144-1152.

29. Tang EH, Leung FP, Huang Y, Feletou M, So KF, et al. (2007) Calcium and reactive oxygen species increase in endothelial cells in response to releasers of endothelium-derived contracting factor. $\mathrm{Br} \mathrm{J}$ Pharmacol 151: 15-23.

30. Griendling KK, Minieri CA, Ollerenshaw JD, Alexander RW (1994) Angiotensin II stimulates $\mathrm{NADH}$ and NADPH oxidase activity in cultured vascular smooth muscle cells. Circ Res 74: 1141-1148.

31. Griendling KK, Sorescu D, Ushio-Fukai M (2000) NAD(P)H oxidase: role in cardiovascular biology and disease. Circ Res 86: 494-501.

32. Toda N, Nakanishi-Toda M (2011) How mental stress affects endothelial function. Pflugers Arch 462: 779-794.

33. Chen H, Zhang L, Zhang M, Song X, Zhang H, et al. (2013) Relationship of depression, stress and endothelial function in stable angina patients. Physio Behav 118: 152-158.

34. Isingrini E, Surget A, Belzung C, Freslon JL, Frisbee J, et al. (2011) Altered aortic vascular reactivity in the unpredictable chronic mild stress model of depression in mice: UCMS causes relaxation impairment to ACh. Physio Behav 103: 540-546.

35. Neves VJ, Moura MJ, Almeida BS, Costa R, Sanches A, et al. (2012) Chronic stress, but not hypercaloric diet, impairs vascular function in rats. Stress 15 138-148.

36. Neves VJ, Moura MJCS, Tamascia ML, Ferreira R, Silva NS, et al. (2009) Proatherosclerotic effects of chronic stress in male rats: Altered phenylephrine sensitivity and nitric oxide synthase activity of aorta and circulating lipids. Stress 12: $320-327$

37. 37.Carda APP, Marchi KC, Rizzi E, Mecawi AS, Antunes-Rodrigues J, et al. (2015) Acute restraint stress induces endothelial dysfunction: role of vasoconstrictor prostanoids and oxidative stress. Stress 18: 233-243.

38. Balkaya M, Prinz V, Custodis F, Gertz K, Kronenberg G, et al. (2011) Stress worsens endothelial function and ischemic stroke via glucocorticoids. Stroke 42: 3258-3264.

39. Huang CJ, Webb HE, Evans RK, McCleod KA, Tangsilsat SE, et al. (2010) Psychological stress during exercise: immunoendocrine and oxidative responses. Exp Biol Med (Maywood) 235: 1498-1504.

40. Groeschel M, Braam B (2011) Connecting chronic and recurrent stress to vascular dysfunction: no relaxed role for the renin-angiotensin system. Am J Physiol Renal Physiol 300: F1-10.

41. Saavedra JM, Benicky J (2007) Brain and peripheral angiotensin II play a major role in stress. Stress 10: 185-193.

42. Clamage DM, Vander AJ, Mouw DR (1977) Psychosocial stimuli and human plasma renin activity. Psychosom Med 39: 393-401.

43. Kosunen KJ, Pakarinen AJ, Kuoppasalmi K, Adlercreutz H (1976) Plasma renin activity, angiotensin II, and aldosterone during intense heat stress. J App Physiol 41: 323-327.

44. Aguilera G, Kiss A, Sunar-Akbasak B (1995) Hyperreninemic hypoaldosteronism after chronic stress in the rat. J Clin Invest 96: 1512-1519.

45. Chung IM, Kim YM, Yoo MH, Shin MK, Kim CK, et al. (2010) Immobilization stress induces endothelial dysfunction by oxidative stress via the activation of the angiotensin II/its type I receptor pathway. Atherosclerosis 213: 109-114.

46. Chies AB, Corrêa FM, de Andrade CR, Rosa-e-Silva AA, Pereira FC, et al (2003) Vascular non-endothelial nitric oxide induced by swimming exercise stress in rats. Clin Exp Pharmacol Physiol 30: 951-957.

47. Bayramgurler D, Karson A, Yazir Y, Celikyurt IK, Kurnaz S, et al. (2013) The effect of etanercept on aortic nitric oxide-dependent vasorelaxation in an unpredictable chronic, mild stress model of depression in rats. Eur J Pharmaco 710: $67-72$.

48. Huang CJ, Webb HE, Zourdos MC, Acevedo EO (2013) Cardiovascular reactivity, stress, and physical activity. Front Physiol 4: 314

49. Choudhury RP, Fuster V, Fayad ZA (2004) Molecular, cellular and functional imaging of atherothrombosis. Nat Rev Drug Discov 3: 913-925. 
Citation: Côco H, Oliveira AM (2015) Endothelial Dysfunction Induced by Chronic Psychological Stress: A Risk Factor for Atherosclerosis. Cardiovasc Pharm Open Access 4: 168. doi:10.4172/2329-6607.1000168

50. O'Leary DH, Polak JF, Kronmal RA, Manolio TA, Burke GL, et al. (1999) Carotid-artery intima and media thickness as a risk factor for myocardial infarction and stroke in older adults. Cardiovascular Health Study Collaborative Research Group. N Engl J Med 340: 14-22.

51. Andersson IJ, Sankaralingam S, Davidge ST (2010) Restraint stress upregulates lectin-like oxidized low-density lipoprotein receptor-1 in aorta of apolipoprotein E-deficient mice. Stress 13: 454-460.

52. Devaki M, Nirupama R, Yajurvedi HN (2013) Chronic stress-induced oxidative damage and hyperlipidemia are accompanied by atherosclerotic development in rats. Stress 16: 233-243.

53. Libby $P$ (2002) Inflammation in atherosclerosis. Nature 420: 868-874.

54. Zhang T, Chen Y, Liu H, Zhou Z, Zhai Y, et al. (2010) Chronic unpredictable stress accelerates atherosclerosis through promoting inflammation in apolipoprotein E knockout mice. Thromb Res 126: 386-392.
55. Zhang T, Zhai Y, Chen Y, Zhou Z, Yang J, et al. (2011) Effects of emotional and physiological stress on plaque instability in apolipoprotein $E$ knockout mice. $J$ Physiol Biochem 67: 401-413.

56. Bernberg E, Ulleryd MA, Johansson ME, Bergström GM (2012) Social disruption stress increases IL-6 levels and accelerates atherosclerosis in ApoE-/- mice. Atherosclerosis 221: 359-365.

57. Lu XT, Liu YF, Zhao L, Li WJ, Yang RX, et al. (2013) Chronic psychological stress induces vascular inflammation in rabbits. Stress 16: 87-98.

58. Djuric Z, Kashif M, Fleming T, Muhammad S, Piel D, et al. (2012) Targeting activation of specific NF-îं $\mathrm{B}$ subunits prevents stress-dependent atherothrombotic gene expression. Mol Med 18: 1375-1386.

59. Heidt T, Sager HB, Courties G, Dutta P, Iwamoto Y, et al. (2014) Chronic variable stress activates hematopoietic stem cells. Nat Med 20: 754-758. 